\title{
Research on a Multi Node Cooperate Image Compression Algorithm for Wireless Network Based on LBT Model
}

\author{
Li Haitao*
}

Shangqiu Normal University, College of Computer and Information Technology, Shangqiu 476000, China

\begin{abstract}
In this paper, we proposed a new multi node cooperate image compression algorithm for wireless network based on LBT model. Firstly, the visual words of query image are built by using the K-means clustering method after the color features and SIFT features of query image are extracted. Then, the visual-words histogram of the query image is carried out by the mobile agent. The image similarity in each node is measured by the Euclidean distance. At last, experiments were adopted to emulate and validate standard images. Results of experiments show that the compression rate of images has been improved within a large compression scope.
\end{abstract}

Keywords: Image compression algorithm, LBT model, multi node cooperate, wireless network.

\section{INTRODUCTION}

As a special application and extension of the traditional Wireless Sensor Networks, Wireless Multimedia Sensor Networks (WMSNs) expand human's horizon to any physical space. It has shown its potential in many areas, such as future information warfare, space warfare, and unmanned combat and civil applications. Besides, the common features of the traditional Wireless Sensor Networks, WMSNs have many unique characteristics, such as high computational complexity and equal energy consumption of image acquisition, computation and transmission. Because of these characteristics, solving the problem of high energy and high cost and prolonging the lifetime of WMSNs becomes the key factor in the practical process of WMSNs [1,2].

WMSN is an advanced form and important branch of WSN. It imports images, audios, videos, sounds and other multimedia information-aware processing functions on the basis of traditional sensor networks. WMSN combines with WSN and multimedia processing technology. It has broad application prospects in real-time monitoring, national defense, military affair, commerce, civil field and so on [3].

Because it can capture images, audios, videos, sounds and other multimedia informations, WMSN also brings rich awareness information [4], but also increases the the complexity of network processing and transmission tasks. Therefore, it is necessary to develop research on high-efficient multimedia information transmission technology in WMSN.

WMSN is widely used in environmental monitoring, battlefield surveillance, traffic monitoring, etc. [5]. Besides selforganizing, unattended it also combines perception of mediarich multimedia technology. On the one hand, it has traditional self-organizing, multi-hop routing, resource constraints and other common characteristics. On the other hand,

\footnotetext{
*Address correspondence to this author at the Shangqiu Normal University, College of Computer and Information Technology, Shangqiu 476000, China; Tel: 15203706708; Fax:0370-2587829; E-mail: Lhtao425@126.com
}

the energy distribution, QoS requirements, sensor model and also has other significant personal characteristics. As energy of nodes, processing power, and storage capacity of WMSNs is very limited, efficient use of energy is the primary objective of WMSNs for which the compression and transmission of image is critical.

Compression, filtering, and data fusion of information processing technology are widely used in WSNs. Keeping in view the limited energy of single node, i.e. processing power, we can use the processing power of multiple nodes, "within network computing" to complete the compression of multimedia information and upload the small amount of data with sufficient information to the next target step by step [6]. Because of the special requirements, based on the improvement of existing algorithms, a distributed image compression algorithm is proposed.

In WMSNs, the amount of image data gathered by camera nodes is very large. They need to be compressed and processed before transmission. But the memory, computation and energy limitation of individual sensor nodes become a hindrance. In order to overcome the low-power and high image quality requirements of WMSN [7], this paper studies the Lapped Biorthogonal Transform (LBT) theory and proposes a distributed image compression algorithm based on LBT in WMSNs. In this algorithm, based on the clustering architecture, multi-nodes sharing the compression task of image compression and transmission are discussed. Simulation results show that this distributed algorithm can greatly reduce the hardware cost and the energy consumptions in WMSNs. The lifetime of the WMSNs can also be prolonged many times in the case of the sensor nodes deployed densely [8].

\section{THE FRAMEWORK OF MULTI NODES COOPER- ATE IMAGE COMPRESSION}

The image data occupy memory space and a large number of transmission bandwidth. Therefore, image compres- 
sion is necessary, which includes loss compression and lossless compression. The former compression permits some differences existing in the image before compression and after compression, and it is widely applied to landscape and people images; the compression ratio is as high as 40 to 1 . For the human eye is not sensitive to these difference, the original image can be replaced by some approximations, which reflect the compromise deal with accuracy of image data, storage space and bandwidth. The latter compression requires that the image before and after compression are exactly the same. And, it is used for certification signature, file signature images and medical images to avoid wrong judgment; the compression ratio is as low as 2 to 1 .

For the correlation exists widely among adjacent pixels and different color components, the image data include high space redundancy. Therefore, the compression ratio should be obtained as high as possible based on good image's visual effect.

The image compression processor includes three parts: transformer, quantizes and coder. The first part reflects the correspondence between original images and transformed images. The image data representation for more easily compressed is provided by removing redundancy in image data. The second part processes the transformed image data into symbols at finite length, i.e. the irreversible process maps the multi-objects into one-target, which results in the loss of image information directly. The third part codes the transformed coefficients into fixed length or variable length. The latter way called entropy code too, which denotes the transformed image coefficients distribution as a shorter discrete random process.

A multi-nodes image retrieval method is divided into three parts, including visual words extraction, multi-nodes retrieval and relevance feedback. The overall diagram is shown in Fig. (1).

SIFT feature is a kind of local characteristics, so the SIFT features and color features are extracted from image and kmeans clustering algorithm is used to construct image visual words library. Fig. (2) shows the construction processing of visual words.

1) Scale Invariant Feature (SIFT) Extraction: SIFT is a kind of local image descriptor. It has certain robustness for the scale, rotation, translation invariance, illumination changes and three-dimensional projection transformation. It can solve a variety of geometric transformations such as; distortion, affine transform, camera angle transformation, brightness transform, and noise. SIFT feature extraction process includes the following four steps:

Step1: Detection of Scale-Space Extreme. The image is convolved with Gaussian filters at different scales, and then the difference of successive Gaussian-blurred images is taken. Key points are then taken as maxima/minima of the Difference of Gaussians (DoG) that occur at multiple scales. Specifically, a DoG image $D(x, y, \sigma)$ is given by,

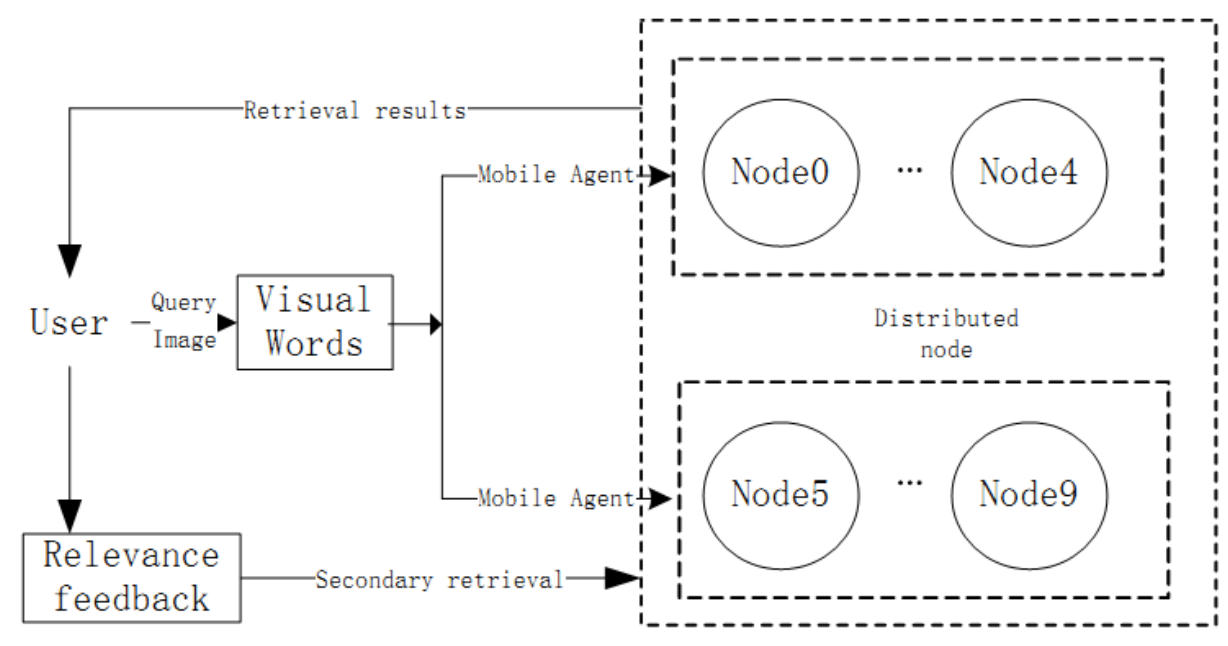

Fig. (1). The proposed multi-nodes image compression diagram.

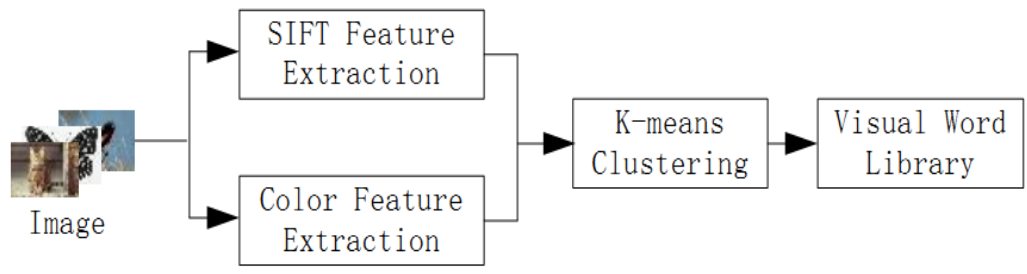

Fig. (2). The visual words library construction. 
$D(x, y, \sigma)=L(x, y, k \sigma)-L(x, y, \sigma)$

Where $L(x, y, \sigma)$ is the convolution of the original image $I(x, y)$ with the Gaussian blur $G(x, y, \sigma)$ at scale $k \sigma, \mathrm{k}$ is a constant multiplicative factor and we have:

$L(x, y, k \sigma)=G(x, y, k \sigma) * I(x, y)$

Step 2: Accurate Key points Localization. Key points are identified as local minima/maxima of the DoG images across scales. This is done by comparing each pixel in the DoG images to its eight neighbors at the same scale and nine corresponding neighboring pixels in each of the neighboring scales. Then used the (3) to calculate the $\mathrm{D}(\mathrm{X})$ of these key points and established the extreme value that is greater than 0.03 as the candidate key.

$D(X)=D+\frac{\partial D^{T}}{\partial X} X+\frac{1}{2} X^{T} \frac{\partial^{2} D^{T}}{\partial X^{2}} X$

Where D and its derivatives are evaluated at the candidate key point and $X=(x, y, \sigma)$ is the offset from this point. Finally, in order to increase stability, the Hessian matrix is used to remove the unstable edge response points.

Step 3: Orientation Assignment. In this step, according to the scale of the key point and the location information, the direction of the coefficient is calculated using the following formulas:

$D(X)=D+\frac{\partial D^{T}}{\partial X} X+\frac{1}{2} X^{T} \frac{\partial^{2} D^{T}}{\partial X^{2}} X$

$m(x, y)=\sqrt{\begin{array}{l}(L(x+1), y)-L(x-1, y)^{2} \\ +(L(x, y+1)-L(x, y-1))^{2}\end{array}}$

$\theta(x, y)=\tan ^{-1} \frac{L(x, y+1)-L(x, y-1)}{(L(x+1), y)-L(x-1, y)}$

Step 4: The Local Image Descriptor. The magnitude and direction calculations for the gradient are done for every pixel in the neighboring region around the key point. An orientation histogram with 36 bins is formed, with each bin covering 10 degrees. A set of orientation histograms is created on $4 \times 4$ pixel neighborhoods with 8 bins each. These histograms are computed from magnitude and orientation values of samples in a $16 \times 16$ region around the key point. Since there are $4 \times 4=16$ histograms each with 8 bins, the vector has 128 dimensions. Color is the most important image feature. HSV space is a color model that is suitable for the human visual system, which is widely used in the computer vision field. It has three attributes: Hue $(\mathrm{H})$, Saturation $(\mathrm{S})$, and Value (V).

Because the human eye's ability to distinguish colors is limited, the color space quantization of non-equal spacing is adopted. Firstly, the RGB color space is converted into the HSV color space. Then, we quantize the color space into non-equidistant according to the color perception of human visual system.
In this paper, we use K-means clustering to analyze the SIFT and HSV color features of image, each cluster center represents a visual word. In the experiment, we select the 500 cluster centers. Fig. (3) depicts query image and the histogram of its visual words, whereas the horizontal axis represents the number of visual words; and the vertical axis represents the weight of the visual words.
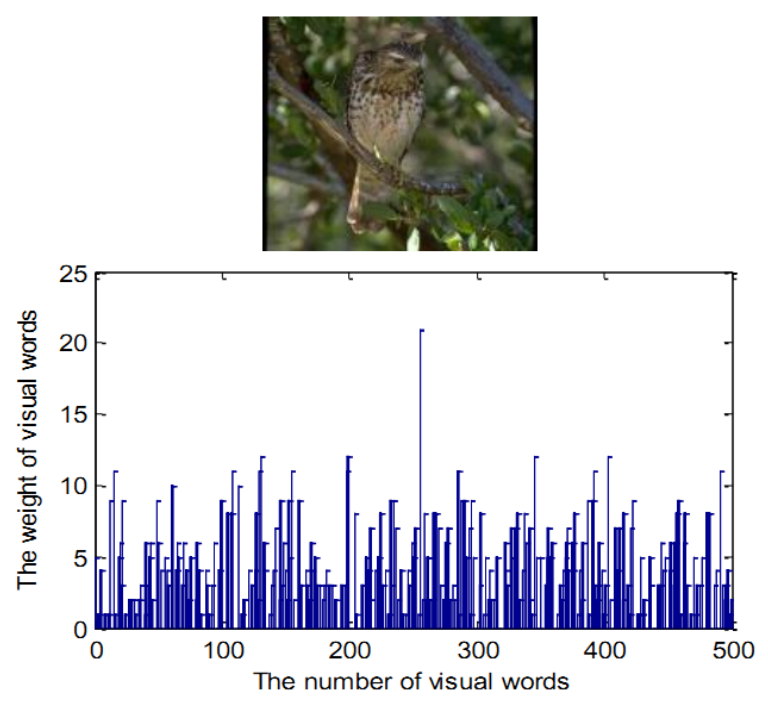

Fig. (3). The query image and the histogram of its visual words.

$\mathrm{K}$-means clustering is a kind of unsupervised learning algorithm. The data set and cluster number $\mathrm{K}$ are given. The clustering process is as follows: $\mathrm{K}$ data points from the data set are chosen as the initial clustering centers.

The clustering centers are recalculated according to the distance among the initial clustering centers and the rest of the data.

The objective function is calculated to judge whether to end the iterative process. In this paper, the SIFT and color feature are respectively calculated according to the K-means clustering and each cluster center respectively includes 128 and 72 dimensions. Then, the TF-IDF is calculated to build the histogram of visual words.

In this paper, the multi-nodes processing diagram is shown in the Fig. (4). The number of images in each node is equal. The multithreaded way is used to achieve the mobile Agent based image retrieval in this paper. In addition, two threads are used to implement the image retrieval each of which manages five nodes.

When the program starts running, the feature of query image is extracted by the mobile Agent, the similarity between the query image and the node image database are measured according to the Euclidean distance, and the results are based on this similarity. Three images with minimum distance and their address information of each node are returned. The returned images of all nodes need to be ordered. Nine images with minimum distance are returned to the user. 


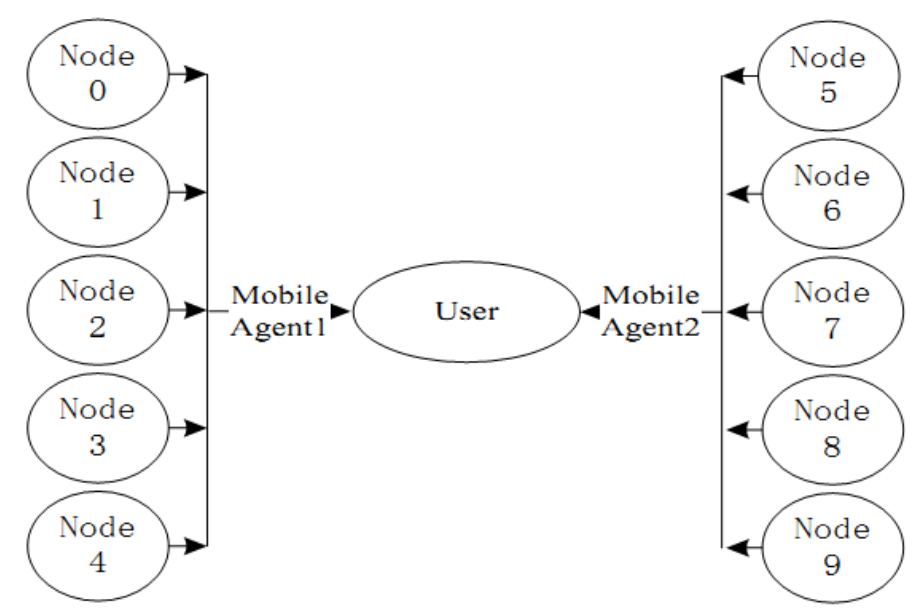

Fig. (4). The diagram of multi-node processing.

The retrieval results are evaluated according to the users' subjective degree. Then, the weight of the characteristics of the node whose image is evaluated, is adjusted according to the users' evaluation.

\section{THE IMPROVEMENT OF IMAGE COMPRESSION ON THE BASIS OF LBT MODEL}

For the purpose of maximizing the lifetime of the network while still ensuring the monitoring quality, a two-layer cooperative WMSNs architecture is proposed. Based on the three-level cooperation model between the camera-equipped node layer and the normal sensor node layer, the main design idea of the proposed architecture is to share the cameraequipped node's energy consumption burden of image acquisition and compression with the normal sensor nodes which are deployed densely. The proposed network architecture is low-cost, and suitable for large-scale deployment.

Firstly, divide $\mathrm{x}$ and $\mathrm{y}$ into blocks by the periodicity of cosine function which is featured by its periodicity. Analysis is made using the periodicity and symmetry of function. When the value of $U$ and $V$ is certain, substitute the value of $\mathrm{X}$ and $\mathrm{Y}$ into cosine formula; when $\mathrm{x}=0$ and $\mathrm{x}=16$, the value of the function is the same and their period is $2 \pi$. According to this rule, we divide $\mathrm{x}$ and $\mathrm{y}$ into blocks within $0-255$ and get 15 blocks shown in Table $\mathbf{I}$. When $\mathrm{x}=94$, we can see from Table I that it is K14. In this way, we can reduce the calculation involving 256 functions to the one with only 16 functions, greatly decreasing the calculation volume of computer.

Secondly, it is the creation of cosine coefficient table. In the above, we grouped the 256 sets of $x$ and $y$ values by their periodicity and 16 groups were obtained from 0 to 15 . As the 8-bit plane image is a 2-D data, make the $16 \mathrm{x}$-y blocks into an address mapping table of 2-D cosine coefficients based on the original block, as shown in Table II. Then, when $\mathrm{x}=0$, $y=0$, we can get $K(0,0)$ from Table II. Likewise, when $x=66$, $\mathrm{y}=100$, Table 1 shows that $\mathrm{x}$ is K2, and y being k4. From Table II, we can find that cosine mapping coefficient is (2, 4).
At last, cosine values were created according to mapping table of cosine coefficients. In the above, we only got the mapping value of coefficients of cosine function by referring to the table according to values of $\mathrm{x}$ and $\mathrm{y}$. In practical technology, what is required is the value of each cosine coefficient, but not the coefficient itself, when $\mathrm{u}, \mathrm{v}, \mathrm{x}$ and $\mathrm{y}$ are given different values in the formula 2. Therefore, in each block of Table II, we substituted the value of v and u varying within $0 \sim 7$ for the coefficients for calculation and obtained the mapping table of practical coefficient values e.g. Table III. For example, in coefficient $\mathrm{K}(0,0)$, as the values of $\mathrm{x}$ and $\mathrm{y}$ are constant, the coefficients of formula 2 are the 8 numbers when $\mathrm{u}$ and $\mathrm{v}$ are assigned $0 \sim 7$, so we have a table of $8 * 8=64$ cosine function values, as shown in Table III.

Table II shows that each block requires a cosine value table, so $15 * 15=256$ tables of each are required as well as $64 * 256$ operations, which is lesser compared with the $8 * 8 * 256 * 256$ cosine operations mentioned above.

For calculation, first multiply each LBT coefficient by $1 / 4$ and possible value of image data varying within $0 \sim 255$. Check the block value from Table $\mathbf{I}$ and then the value of LBT coefficient from Table II with block value. From Table II, we find that the value of $u$ and $v$ determines which coefficient of cosine terms to be multiplied with $f(x, y)$. By this relation, we can make an address mapping table to check related coefficients in LBT transformation. At the start of LBT transformation, the system initially loads the coefficient table and address mapping table, then determines the position in address mapping table by the value of $\mathrm{u}$ and $\mathrm{v}$, and draws the 64 values from the back, and locates the starting place of these values in coefficient table, and adds the value of $f(x, y)$, and then adds the values to finish the calculation of one system in LBT. The above shows that in applying table inquiry method, the system multiplies the coefficients with all possible values of image, and then saves the results in the table which is loaded into the memory during operation. This way the operation of multiplication can be avoided, thereby accelerating the operation rate compared to regular algorithms. 
Table I. The block table of $\mathrm{X}$ and $\mathrm{Y}$.

\begin{tabular}{|l|l|l|l|l|l|l|l|l|l|}
\hline K0 & K1 & K2 & K3 & K4 & K5 & K6 & $\ldots$ & K14 & K15 \\
\hline 0 & 1 & 2 & 3 & 4 & 5 & 6 & $\ldots$ & 14 & 15 \\
\hline 16 & 17 & 18 & 19 & 20 & 21 & 22 & $\ldots$ & 30 & 31 \\
\hline 32 & 33 & 34 & 35 & 36 & 37 & 38 & $\ldots$ & 46 & 47 \\
\hline 48 & 49 & 50 & 51 & 52 & 53 & 54 & $\ldots$ & & 63 \\
\hline 64 & 65 & 66 & 67 & 68 & 69 & 70 & $\ldots$ & 78 & 79 \\
\hline 80 & 81 & 82 & 83 & 84 & 85 & 86 & $\ldots$ & 94 & 95 \\
\hline 96 & 97 & 98 & 99 & 100 & 101 & 102 & $\ldots$ & 110 & 111 \\
\hline$\ldots$ & $\ldots$ & $\ldots$ & $\ldots$ & $\ldots$ & $\ldots$ & $\ldots$ & $\ldots$ & $\ldots$ & $\ldots$ \\
\hline 240 & 241 & 242 & 243 & 244 & 245 & 246 & $\ldots$ & 254 & 255 \\
\hline
\end{tabular}

Table II. The address mapping table of X-Y cosine address.

\begin{tabular}{|c|c|c|c|c|c|c|}
\hline & 0 & 1 & 2 & 3 & $\ldots$ & 15 \\
\hline 0 & $\mathrm{~K}(0,0)$ & $\mathrm{K}(0,1)$ & $\mathrm{K}(0,2)$ & $\mathrm{K}(0,3)$ & $\ldots$ & $\mathrm{K}(0,15)$ \\
\hline 1 & $\mathrm{~K}(1,0)$ & $\mathrm{K}(1,1)$ & $\mathrm{K}(1,2)$ & $\mathrm{K}(1,3)$ & $\ldots$ & $\mathrm{K}(0,15)$ \\
\hline 2 & $\mathrm{~K}(2,0)$ & $\mathrm{K}(2,0)$ & $\mathrm{K}(2,0)$ & $\mathrm{K}(2,0)$ & $\ldots$ & $\mathrm{K}(0,15)$ \\
\hline 3 & $\mathrm{~K}(3,0)$ & $\mathrm{K}(3,1)$ & $\mathrm{K}(3,2)$ & $\mathrm{K}(3,3)$ & $\ldots$ & $\mathrm{K}(0,15)$ \\
\hline 4 & $\mathrm{~K}(4,0)$ & $\mathrm{K}(4,1)$ & $\mathrm{K}(4,2)$ & $\mathrm{K}(4,3)$ & $\ldots$ & $\mathrm{K}(0,15)$ \\
\hline$\ldots$ & $\ldots$ & $\ldots$ & $\ldots$ & $\ldots$ & $\ldots$ & \\
\hline 15 & $\mathrm{~K}(15,0)$ & $\mathrm{K}(15,1)$ & $\mathrm{K}(15,2)$ & $\mathrm{K}(15,3)$ & $\ldots$ & $\mathrm{K}(15,15)$ \\
\hline
\end{tabular}

Table III. The X-Y cosine function value.

\begin{tabular}{|c|c|c|c|c|c|c|c|c|}
\hline & 0 & 1 & 2 & 3 & 4 & 5 & 6 & 7 \\
\hline 0 & $\mathrm{C} 0 * \mathrm{C} 0$ & $\mathrm{C} 1 * \mathrm{C} 0$ & $\mathrm{C} 2 * \mathrm{C} 0$ & $\mathrm{C} 3 * \mathrm{C} 0$ & $\mathrm{C} 4 * \mathrm{C} 0$ & $\mathrm{C} 5 * \mathrm{C} 0$ & $\mathrm{C} 6 * \mathrm{C} 0$ & $\mathrm{C} 7 * \mathrm{C} 0$ \\
\hline 1 & $\mathrm{C} 0 * \mathrm{C} 1$ & $\mathrm{C} 1 * \mathrm{C} 1$ & $\mathrm{C} 2 * \mathrm{C} 1$ & $\mathrm{C} 3 * \mathrm{C} 1$ & $\mathrm{C} 4 * \mathrm{C} 1$ & $\mathrm{C} 5 * \mathrm{C} 1$ & $\mathrm{C} 6 * \mathrm{C} 1$ & $\mathrm{C} 7 * \mathrm{C} 1$ \\
\hline 2 & $\mathrm{C} 0 * \mathrm{C} 2$ & $\mathrm{C} 1 * \mathrm{C} 2$ & $\mathrm{C} 2 * \mathrm{C} 2$ & $\mathrm{C} 3 * \mathrm{C} 2$ & $\mathrm{C} 4 * \mathrm{C} 2$ & $\mathrm{C} 5 * \mathrm{C} 2$ & C6*C2 & $\mathrm{C} 7 * \mathrm{C} 2$ \\
\hline 3 & $\mathrm{C} 0 * \mathrm{C} 3$ & $\mathrm{C} 1 * \mathrm{C} 3$ & $\mathrm{C} 2 * \mathrm{C} 3$ & $\mathrm{C} 3 * \mathrm{C} 3$ & $\mathrm{C} 4 * \mathrm{C} 3$ & $\mathrm{C} 5 * \mathrm{C} 3$ & $\mathrm{C} 6 * \mathrm{C} 3$ & $\mathrm{C} 7 * \mathrm{C} 3$ \\
\hline 4 & $\mathrm{C} 0 * \mathrm{C} 4$ & $\mathrm{C} 1 * \mathrm{C} 4$ & $\mathrm{C} 2 * \mathrm{C} 4$ & $\mathrm{C} 3 * \mathrm{C} 4$ & $\mathrm{C} 4 * \mathrm{C} 4$ & $\mathrm{C} 5 * \mathrm{C} 4$ & $\mathrm{C} 6 * \mathrm{C} 4$ & $\mathrm{C} 7 * \mathrm{C} 4$ \\
\hline 5 & $\mathrm{C} 0 * \mathrm{C} 5$ & $\mathrm{C} 1 * \mathrm{C} 5$ & $\mathrm{C} 2 * \mathrm{C} 5$ & $\mathrm{C} 3 * \mathrm{C} 5$ & $\mathrm{C} 4 * \mathrm{C} 5$ & $\mathrm{C} 5 * \mathrm{C} 5$ & $\mathrm{C} 6 * \mathrm{C} 5$ & $\mathrm{C} 7 * \mathrm{C} 5$ \\
\hline 6 & $\mathrm{C} 0 * \mathrm{C} 6$ & $\mathrm{C} 1 * \mathrm{C} 6$ & $\mathrm{C} 2 * \mathrm{C} 6$ & C3*C6 & $\mathrm{C} 4 * \mathrm{C} 6$ & $\mathrm{C} 5 * \mathrm{C} 6$ & C6*C6 & $\mathrm{C} 7 * \mathrm{C} 6$ \\
\hline 7 & $\mathrm{C} 0 * \mathrm{C} 7$ & $\mathrm{C} 1 * \mathrm{C} 7$ & $\mathrm{C} 2 * \mathrm{C} 7$ & C3*C7 & $\mathrm{C} 4 * \mathrm{C} 7$ & $\mathrm{C} 5 * \mathrm{C} 7$ & $\mathrm{C} 6 * \mathrm{C} 7$ & $\mathrm{C} 7 * \mathrm{C} 7$ \\
\hline
\end{tabular}

To make an intuitionistic comparison, we performed a simulation experiment of JPEG standard algorithm and improved LBT algorithm with two images in the same computer. Coding time was calculated by setting time parameter and the result is shown in Table IV. This experiment adopted images sorce1.bmp and sorce2.bmp, which are $256 * 256$ and 8-bit respectively. Experimental results show that the improved algorithm raised by this paper saves about $40 \%$ of time compared to original algorithm.

Table IV. The comparison of operation rate.

\begin{tabular}{|l|l|l|}
\hline & $\begin{array}{l}\text { Original } \\
\text { Algorithm }\end{array}$ & $\begin{array}{l}\text { Improved } \\
\text { Algorithm }\end{array}$ \\
\hline Sorce $1\left(10^{-6} \mathbf{s}\right)$ & 5.632 & 3.168 \\
\hline Sorce1 $\left(10^{-6} \mathbf{s}\right)$ & 7.426 & 4.502 \\
\hline
\end{tabular}

\section{CONCLUSION}

In view of low compression rate of JPEG algorithm caused by the large amount of cosine calculation, we created the table of a series of cosine values by periodicity of cosine function, and then carried out LBT transformation through table reference. This has greatly reduced large amount of cosine operation improving the compression rate of JPEG. An analysis was made based on experiments. Many points need to be improved in the process, such as, the compression effect on larger images is imperfect. It is expected to improve the compression quality of various sizes of images by modifying the quantization process. We can still take advantage of improved multimedia performance of computers to divide the conventional $8 * 8$ data block to $16 * 16$ one. 


\section{CONFLICT OF INTEREST}

The authors confirm that this article content has no conflict of interest.

\section{ACKNOWLEDGEMENTS}

This work is supported by the Young Teachers Subsidy Scheme in 2013 of the Shangqiu Normal University, China (no. 2013GGJS12), and the basic and frontier technology research projects of Henan Province, China (no. 132300410203).

\section{REFERENCES}

[1] J. Zhang, L.Z. Cheng, H.B. Yang, and K.H. Huang, "Adaptive Lifting Wavelet Transform and Image Compression Via Texture," Chinese Journal of Computers, vol. 1, pp.184-192, 2010.
[2] D.L. Donoho, "Compressed sensing," IEEE Transactions on Information Theory, vol. 4, pp. 1289-1306,2006.

[3] E. Candes, J. Romberg, and T. Tao, "Robust uncertainty principles: Exact signal reconstruction from highly incomplete frequency information," IEEE Transactions on Information Theory, vol. 2, pp. 489-509, 2006.

[4] L.B. Joe, "Wavelet analysis and their application in medicine,"Mathematical Medicine, vol. 2, pp. 155 156, 2003.

[5] M.N. Do, and M. Vetterli, "The contourlet transform: An efficient directional multi-resolution image representation," IEEE Transcactions on Image Processing, vol. 12, pp. 2091-2106, 2005.

[6] L. Gan, "Block Compressed Sensing of Natural Images," The 15th International Conference on Digital Signal Processing, pp. 403406, 2007.

[7] Z. Jin, and G. Hu, "Application of inquiry optimized in the Distributed database system," Computer Applications and Software, pp. 58-60, 2013.

[8] P. Vassiliadis, and T. Sellis, "A Survey on Logical Models for OLAP Databases,” ACM SIGMOD Record, vol. 4, pp.64-69, 2009.

Received: September 16, 2014

Revised: December 23, 2014

Accepted: December 31, 2014

(C) Li Haitao; Licensee Bentham Open.

This is an open access articles licensed under the terms of the Creative Commons Attribution-Non-Commercial 4.0 International Public License (CC BY-NC 4.0) (https://creativecommons.org/licenses/by-nc/4.0/legalcode), which permits unrestricted, non-commercial use, distribution and reproduction in any medium, provided that the work is properly cited. 